\title{
PERCEPCIÓN DEL ESTUDIANTE DE ENFERMERÍA DE LA U.D.C.A FRENTE A LA FORMACIÓN POR COMPETENCIAS Y LOS RESULTADOS EN LAS PRUEBAS DE EVALUACIÓN
}

\section{PERCEPTION OF U.D.C.A,s NURSING STUDENTS TOWARD THE FORMATION BY COMPETITIONS AND THE RESULTS OF EVALUATION TESTS}

Vilma Cenit Fandiño Osorio Lorena Martínez Delgado ${ }^{2}$

\section{RESUMEN}

En Colombia la formación en la educación se ha reglamentado por competencias y es así que este texto hace referencia a la movilización de saberes y destrezas en diversas áreas que se combinan para resolver problemas y producir nuevos conocimientos. El objetivo fue identificar la percepción del estudiante de Enfermería frente a la formación por competencias, en los programas académicos del área profesional y la correlación existente con el resultado obtenido en los exámenes de calidad del estado (ECAES). Se realizó un estudio descriptivo correlacional y evaluativo en el que se aplicó un instrumento que midió la percepción del estudiante sobre su formación, los resultados de las notas finales de cada uno de los programas académicos de Cuidado de Enfermería y los resultados del ECAES obtenidos por los estudiantes de último año. El análisis, arrojó una diferencia entre el promedio de percepción de los estudiantes sobre los logros alcanzados en los programas académicos y el promedio obtenido en la prueba de competencias, diseñada por ACOFAENICFES. Los estudiantes consideran estar preparados para enfrentar el mundo laboral, aunque en el examen de

1 Enfermera. Especialista en Gerencia Educativa. Magister con énfasis en Cuidado Materno. Facultad de Enfermería. Universidad de Ciencias Aplicadas y Ambientales U.D.C.A. e-mail: vfandino@udca.edu.co

2 Enfermera. Especialista en Docencia Universitaria y en Educación para la Salud. Facultad de Enfermería. Universidad de Ciencias Aplicadas y Ambientales U.D.C.A. e-mail: lorenamartinez53@hotmail.com. valoración, por competencias de la educación superior, el promedio no corresponde a la apreciación perceptiva de cumplimiento de logro. Se encontró una relación directamente proporcional entre el promedio general del estudiante y el puntaje de ECAES. Es así, como los estudiantes con altos promedios en la Facultad también mostraron altos puntajes en la prueba de estado.

Palabras clave: Percepción, enfermería, competencias.

\section{SUMMARY}

In Colombia the educational formation has been regulated by competitions and this context makes reference to the mobilization of knowledge and skills in diverse areas that are combined to solve problems and to produce new wisdom. The objective of this research was to identify the perception of the infirmary student, in front of the formation by competitions in the academic programs of the professional area and the existing correlation with the result obtained in the state qualification examinations (ECAES). A co relational and evaluative descriptive study, in which an instrument was applied that measured the perception of the student regarding its formation, the results of final notes of each of the academic programs denominated "Infirmary Care Taking" and the results of the ECAES obtained by the students of the last academic year. The data analysis showed differences between the average perception of the students about the grades reached within the Academic Programs and the average obtained in the test of competitions designed by 
ACOFAEN-ICFES. The students consider to be prepared to face the labour world, although in the examination of valuation by competitions of the higher education, the average obtained did not correspond to the perceptive valuation of fulfilment. A direct proportional relation between the general average of the students and the points of ECAES was found. Thus, students with high averages in the faculty also showed high points in the state qualification examination.

Key words: Perception, infirmary, competitions.

\section{INTRODUCCIÓN}

Este artículo toma como referente los resultados obtenidos en la investigación sobre formación por competencias en la Facultad de Enfermería de la Universidad de Ciencias Aplicadas y Ambientales U.D.C.A, relacionados con la percepción del estudiante frente al logro de competencias en el área de formación profesional y su relación con en el ECAES.

Educación por competencias: El discurso sobre competencias, que no es nuevo en nuestro país, es un discurso que se ha convertido en una de las principales preocupaciones de las Instituciones Educativas. De la misma forma, se considera un tema relevante dentro de una sociedad, que se replantea a sí misma la relación estrecha entre la calidad de la educación, el mundo laboral, el mundo educativo y el mundo de la globalización.

Colombia ha estado muy atenta a las recomendaciones internacionales y a los diagnósticos nacionales en el tema del mejoramiento de la calidad de la educación. Las políticas en marcha (Ley 30 de 1992, ley 115 de 1994, decreto 917 del 2001) (REPÚBLICA DE COLOMBIA, 2007), estipulan dentro del contexto de la educación superior, sobre la responsabilidad conjunta entre las instancias para cumplir con unos mínimos estándares que permitan certificar calidad y abrir posibilidades para acreditar programas de manera voluntaria, situación que se enmarca dentro de una discusión sobre las competencias que se deben generar en un profesional para que responda a las necesidades de un país y sea capaz de competir dentro del contexto que impone el proceso de globalización.

Exámenes de calidad de la educación superior: El ICFES, comprometido con el logro del mejoramiento de la calidad de la Educación Superior y en cumplimiento de la Política "Por una Educación Superior Responsable y de Excelencia", planteó la necesidad de un SISTEMA de evaluación bien diseñado e instrumentado.

Los Exámenes de Calidad de la Educación Superior (ECAES) tienen como propósito para Enfermería indagar sobre el desarrollo de las competencias básicas y fundamentales del profesional de esta disciplina, de acuerdo con el perfil profesional que, por consenso, se ha definido y que surge del proceso de análisis curricular (ACOFAEN, 2003).

Algunos de los factores identificados por ACOFAEN, como elementos que se deben tener en cuenta en la preparación de los estudiantes para la presentación de los ECAES, que influyen y determinan la calidad de la educación son: los que afectan el proceso de aprendizaje (tiempo efectivo de clases, acceso a material didáctico, calidad de los profesores, pertinencia del currículo, gestión administrativa de la educación, factores de entorno y de contexto social) y la existencia de un compromiso de los diversos agentes sobre la importancia social de la educación, lo cual produce su participación activa y control del sistema y de sus resultados (ACOFAEN, 2005).

Por tanto, el resultado obtenido por los estudiantes en la prueba ECAES, se convierte para cada Facultad en un elemento que retroalimenta y autoevalúa el proceso de calidad y de formación de los estudiantes, medido por las competencias alcanzadas por cada uno de ellos en las áreas de formación básica, socio-humanística y profesional.

Percepción del estudiante: Para determinar la percepción del estudiante de la Facultad de Enfermería frente al logro de competencias en las áreas de profesionalización, se conceptualizó percepción de la siguiente forma:

La percepción ha sido definida como aquella capacidad del ser humano para interpretar la realidad utilizando los órganos de los sentidos. La percepción nunca es igual en cada individuo, cada sujeto construye su realidad desde las experiencias anteriores y desde el contexto que lo rodea, de tal forma que ésta se ve mediada por las características individuales, sociales y culturales de cada sujeto.

Desde la infancia y a través del lenguaje, los individuos utilizando la comunicación y las relaciones interpersonales, aprenden a dar significado y a determinar las cosas que 
son importantes en su vida. Esta idea vista desde los procesos de aprendizaje y empleando el lenguaje, como mediador, ha sido estudiada por diversos autores, entre los cuales figura Cicarelli (2003). Esta autora, citando a Smith afirma que el proceso de aprendizaje consta de tres fases: la percepción, la ideación y la exposición. En la primera, el individuo considera los aspectos singulares de la experiencia; la ideación permite la reflexión sobre la experiencia y, en la exposición, se expresa todo el conocimiento adquirido.

Pero el proceso de aprendizaje es reciproco, no es unidireccional, de tal forma que para que cada estudiante adopte aprendizajes significativos y pueda realizar una construcción propia de conocimiento que no se quede en la percepción de la realidad, el material que presente el docente debe tener significado y llevar una secuencia lógica que permita al estudiante integrar los nuevos conocimientos y relacionarlos con los ya procesados.

Es importante resaltar que el aprendizaje de los estudiantes no se realiza solo desde contenidos teóricos sino que a su vez están implícitos valores, principios y concepciones de la realidad, que forman en ellos unas actitudes y una visión del mundo para interpretar las situaciones a las que se enfrentan.

En Enfermería, específicamente las situaciones de cuidado a las que se enfrenta el estudiante, conllevan no solo una percepción de la realidad sino un análisis de la misma, que le permite identificar las necesidades de los sujetos de atención y planear un cuidado de Enfermería acorde a ésta.

Así mismo, el estudiante de Enfermería debe tener presente que para brindar Cuidado de Enfermería debe entender y contextualizar su sujeto de cuidado, como un individuo que tiene una percepción individual y social de su Salud, determinada por el contexto social y cultural en que se ha desarrollado.

Es así, como la percepción de la realidad para cada sujeto se convierte en una contínua construcción conceptual que organiza la información y se integra con la información sensorial, con nuestros ideales y con lo que cada sujeto considera prioritario. De esta forma, las percepciones en el hombre generan conductas y realidades únicas para cada ser y para cada cuidador.

La práctica de Cuidado en cada etapa del ciclo vital humano, como lo realiza el estudiante durante su etapa de formación, le permite adquirir competencias específicas para la solución de diferentes situaciones de salud.

El objetivo general de este estudio fue identificar la percepción del estudiante de Enfermería de la U.D.C.A frente a la formación por competencias, mediante la determinación de la correlación entre la percepción del estudiante de último año, de los logros alcanzados al final de los programas académicos del área de profesionalización y los Exámenes de Calidad de la Educación Superior (ECAES), estableciendo la relación entre los promedios de calificación general alcanzados por los estudiantes de último año y el puntaje obtenido en el Examen de Calidad de la Educación Superior ECAES.

\section{MATERIALES Y MÉTODOS}

Se tomaron como marco de referencia los propósitos con los que se establecieron los ECAES para los estudiantes de Enfermería y la conceptualización de lo que significa la percepción.

El tipo de estudio fue descriptivo co-relacional y evaluativo, tomando como referente, para la evaluación de las competencias, los programas académicos de Cuidado de Enfermería, correspondientes al ciclo de profesionalización del currículum de la Facultad de Enfermería de la U.D.C.A, primer periodo académico 2007 (Cuidado de Enfermería Materno-Infantil, Cuidado de Enfermería al Niño y al Adolescente, Cuidado de Enfermería al Adulto y al Anciano y Cuidado de Enfermería en Programas Especiales), las notas finales obtenidas por los estudiantes en los diferentes Cuidados de Enfermería y los resultados de los ECAES. Los sujetos de estudio fueron los estudiantes de último año de la Facultad de Enfermería de la U.D.C.A, en el 2007

Las variables y categorías que se establecieron fueron: la formulación de competencias en los programas, la percepción de logro del estudiante frente a las competencias propuestas por el programa, el logro de competencias de los estudiantes frente a los promedios generales de la Facultad y los resultados de ECAES.

En cuanto a la muestra, se analizaron las competencias de los programas académicos de Cuidado de Enfermería con sus respectivas competencias y 18 estudiantes de último semestre de la Facultad. Las competencias por programas académicos, se presentan en la Tabla 1. 
Tabla 1. Programas académicos y competencias. Facultad de Enfermería U.D.C.A .

\section{CUIDADO DE ENFERMERÍA MATERNO INFANTIL}

Realiza idóneamente la consulta prenatal, consejería de planificación, cuidado a la gestante hospitalizada, promoviendo la participación del padre y la familia en todo su proceso, de vida.

Lidera proceso que le permiten que identifique, planee, proponga, desarrolle y evalué, los programa en salud sexual y reproductiva dentro del marco de derechos; con una perspectiva de género, aplicando los conocimientos y técnicas y cumpliendo con las funciones como enfermera(o), que le competen en los programas de planificación familiar, detección precoz de cáncer Cerviñouterino y de mama, educación en el pre, tras y post-parto.

Posee habilidades para desempeñar los roles administrativos, investigativos, asistencial y educativo del profesional de Enfermería en Servicios de Salud, a través del manejo de problemáticas más relevantes en el proceso de salud enfermedad con la población materno-infantil en el ambiente familiar, institucional.

Brinda Cuidado de Enfermería al binomio madre hijo y la familia dentro de la normatividad actual para la atención de la gestación, parto y posparto y los cuidados en puericultura según necesidades detectadas.

Identifica las normas que existen dentro del régimen general de Seguridad Social de Salud en lo relacionado con la Salud Materno Infantil y la salud sexual y reproductiva (POS-POSS -PAB); además de tener en cuenta las reglas y normas institucionales teniendo en cuenta los niveles jerárquicos.

Desarrollar habilidades prácticas y actitudes éticas, que permitan su actuar profesional dentro del área materno-infantil, con base en los conocimientos adquiridos a nivel técnico, científico, legal, laboral y ético, teniendo en cuente el ambiente natural y social donde se desarrollan los individuos.

\section{CUIDADO DE ENFERMERÍA EL NIÑO Y EL ADOLESCENTE}

Describe las políticas y programas relacionados a la atención del niño y del adolescente en Colombia y América Latina.

Relaciona las líneas de acción y estrategias de las políticas y programas en la atención del niño y del adolescente con el entorno donde se encuentra brindando cuidado.

Gestiona los recursos de acuerdo a las prioridades del cuidado.

Reconoce al niño y cuidador como sujeto de cuidado, a través de una relación interpersonal, cercana, cálida y humana.

El estudiante brinda cuidado integral de enfermería al niño y al adolescente aplicando el proceso de enfermería en el marco de referencia de Patrones Funcionales de Salud de Maryory Gordon.

El estudiante realiza el cuidado procedimental con sustento teórico, tanto de anatomofisiológia y psicología, como de teorías y principios de enfermería.

Elabora plan de intervención con la familia o cuidador en pro del desarrollo, cuidado, formación y bienestar del niño y adolescente.

\section{CUIDADO DE ENFERMERÍA AL ADULTO Y EL ANCIANO}

Brindar cuidado al Adulto y al Anciano, mediante la aplicación del proceso de atención de enfermería, basado en referentes teóricos y conceptuales de Dorothea Orem, haciendo énfasis en la valoración por requisitos de Auto-cuidado y abordando al sujeto de cuidado, como una estructura integral y desarrollando su capacidad analítica, que garantice el cuidado a la persona que se encuentra bajo su responsabilidad.

Establecer pautas precisas de atención del adulto y al anciano, teniendo en cuenta sus condiciones psicosociales, culturales, económicas y biológicas, así como las tendencias demográficas y el perfil epidemiológico.

Participar en la planeación, ejecución y lo evaluación de programas y de proyectos de salud a nivel Distrital y Local de tipo comunitario, intra o extra-hospitalario.

Planear ejecutar y evaluar acciones, basadas en la identificación de necesidades del servicio o del programa, tendientes al mejoramiento continuo en la prestación de los servicios que se brindan al individuo, familia y comunidad.

Brindar, cuidado al adulto y al anciano, mediante la aplicación del proceso de atención de enfermería, basado en referentes teóricos y conceptuales de Dorothea Orem, haciendo énfasis en la valoración por requisitos de Auto-cuidado y abordando al sujeto de cuidado, como una estructura integral y desarrollando su capacidad analítica, que garantice el cuidado a la persona que se encuentra bajo su responsabilidad. 
Continuación Tabla 1

\begin{tabular}{|c|}
\hline CUIDADO DE ENFERMERÍA EN PROGRAMAS ESPECIALES \\
\hline $\begin{array}{l}\text { Conoce los conceptos, definiciones y normativos alrededor de la situaciones de salud vigentes en la atención de salud a pacientes } \\
\text { con alteraciones agudas de su estado físico y mental. }\end{array}$ \\
\hline $\begin{array}{l}\text { Reconoce e identifica la normatividad existente con relación a la prestación de los servicios, de acuerdo con el tipo de afiliación } \\
\text { al SGSSS, los planes de beneficios, actividades y procedimientos para la atención de los usuarios que presentan un desvió } \\
\text { repentino del estado de salud físico y mental. }\end{array}$ \\
\hline $\begin{array}{l}\text { Reconoce los elementos básicos del perfil epidemiológico: factores de riesgo, estadísticas de atención de salud a nivel departamental } \\
\text { y nacional, en relación con los eventos más frecuentes considerados en el conjunto de situaciones criticas e imprevistas que } \\
\text { afectan el proceso salud - enfermedad. } \\
\text { La funcionalidad permanente o transitoria y el compromiso de la vida bajo los enfoques biosicosocial, multidisciplinario y de } \\
\text { acuerdo con la complejidad de atención. }\end{array}$ \\
\hline $\begin{array}{l}\text { Conocer la estructura técnica y organizacional de los servicios de urgencias, cuidados intensivos e instituciones de salud mental, } \\
\text { en donde el estudiante identifica su relación con la atención a los pacientes desde el componente administrativo y asistencial } \\
\text { del rol profesional. }\end{array}$ \\
\hline $\begin{array}{l}\text { Conoce los elementos normativos y técnicos del sistema de referencia y contrarreferencia instaurados para garantizar la } \\
\text { oportunidad e integralidad en la atención a los pacientes que tienen alteración en la salud de forma repentina en el contexto } \\
\text { local, departamental y nacional. }\end{array}$ \\
\hline Asocia e integra conocimientos teóricos y prácticos para el abordaje de la atención de los pacientes de los servicios, obje \\
\hline
\end{tabular}

Fuente: Programas Académicos. Facultad de Enfermería, año 2007.

En cuanto a las técnicas e instrumentos de recolección, se revisaron los programas académicos y se tomaron como referencia las competencias establecidas para cada curso académico del componente profesional específico. Se aplicó un instrumento que midió la percepción del estudiante frente a su formación, con una escala de 1 a 5, donde uno es la nota mínima y cinco la máxima; se analizaron los resultados de las calificaciones finales de cada uno de los programas académicos de Cuidado de Enfermería y los resultados obtenidos por los estudiantes de Enfermería de la prueba de estado ECAES.

Los datos obtenidos se trascribieron a Excel versión 2003 y se procesaron en SAS (Sitem Analisis Estatic) Versión 9.1.3 para Windows versión 10.07 (paquete estadístico para ciencias sociales).

\section{RESULTADOS Y DISCUSIÓN}

Correlación entre calificaciones finales de los programas académicos y las calificaciones de las percepciones de los estudiantes sobre el logro de las competencias: En general, no se encontró correlación entre la percepción de los estudiantes sobre el logro de las competencias y los resultados de las calificaciones finales de los programas académicos del área de profesionalización fue de $(r=-0.393, p=0.002)$ (Tabla 2).

En los estudiantes, entre más alta es la percepción de haber logrado la competencia, la nota académica tiende a ser más baja, sienten haber aprendido y tener habilidades, aún cuando sus resultados numéricos de evaluaciones no lo demuestran.

Una explicación a esta situación es que durante el proceso de formación se hace énfasis en diversas actividades orientadas hacia la destreza con actividades determinadas y productos concretos, que hace que el estudiante incremente experiencias ante situaciones características utilizando los conocimientos recibidos, lo que va construyendo una percepción positiva teniendo en cuenta sus logros (Moreno, 2004); así mismo, permite que la autoestima del estudiante refuerce sus condiciones psicológicas y establezca una condición de 
Tabla 2. Correlaciones de Pearson de las notas obtenidas en la Facultad, el puntaje de ECAES y la percepción de los estudiantes frente a su aprendizaje.

\begin{tabular}{|c|c|c|c|}
\hline Correlación de Pearson & Notas Finales Facultad & Puntaje ECAES & Percepción \\
\hline Notas Finales Facultad & 1,00 & $0,688^{\star *}$ & $-0,393$ \\
\hline Punta ECAES & 0,688 & 1,00 & $-0,12$ \\
\hline Percepción & $-0,393$ & $-0,12$ & 1 \\
\hline
\end{tabular}

** Correlación es significativa al nivel 0,01

seguridad frente a nuevas situaciones que requieran una rápida y oportuna acción con bases científicas acertadas constituyéndose en una alta percepción.

Intervalos de confianza al 95\% (Tabla 3). En el análisis, total, se puede señalar que hubo una diferencia entre la percepción de los estudiantes de último semestre sobre el logro de las competencias propuestas por los diferentes programas académicos y el promedio general de los mismos estudiantes que presentaron la prueba de estado (ECAES).

En las evaluaciones escritas, los estudiantes ponen en juicio sus procesos cognitivos de saber (conocer), hacer (actuar) y saber hacer (reflexionar), para realizar una toma de decisión adecuada en la situación que se presenta a resolver. Entra también en cuestionamiento la competencia primaria de lectura y escritura, grado de concentración, estado físico y psicológico de los estudiantes durante la prueba de ECAES (Facultad de Enfermería, 2006).

Correlación entre promedio general de las notas finales de los cursos académicos correspondientes al área de profesional específico de los estudiantes de último semestre y el resultado del ECAES: se encontró una correlación medianamente fuerte entre el promedio general del estudiante y el puntaje de ECAES en los estudiantes en general $(r=0.68, p=0.002)$.

Los estudiantes con altos promedios en las notas finales de los programas académicos, también mostraron altos puntajes de ECAES; así mismo, los estudiantes con bajos y medianos promedios de notas en los programas académicos obtuvieron bajos y medianos promedios en los ECAES.

Un indicador para este resultado es el desempeño que ha tenido el estudiante en su proceso de formación y el tipo de aprendizaje que ha realizado. Cuando el estudiante es capaz de analizar e integrar el conocimiento teórico con el práctico puede responder positivamente ante cualquier tipo de evaluación.

Algunos autores consideran dos tipos de evaluaciones: las internas y las externas. Reciben su nombre según el agente educativo que lo utilice; si la realizan agentes del PEI, se consideran internas y tienen como propósito analizar los resultados alcanzados dentro del programa, tomando como referencia los objetivos y las competencias enunciadas al inicio del proceso, pero si la prueba es realizada por evaluadores externos que no tienen contacto con los agentes educativos internos, con criterios e indicadores propios, este tipo de prueba se denomina de evaluación

Tabla 3. Intervalos de confianza al 95\%.

Descriptive Statistics

\begin{tabular}{|c|c|c|c|c|}
\hline & \multicolumn{2}{|c|}{ Mean } & \multirow{2}{*}{$\begin{array}{l}\text { Límite } \\
\text { Inferior }\end{array}$} & \multirow{2}{*}{$\begin{array}{l}\text { Límite } \\
\text { Superior }\end{array}$} \\
\hline & Statistic & Std. Error & & \\
\hline Facultad & 3,6794 & 0,04734 & 3,58665 & 3,77224 \\
\hline ECAES & 101,661 & 1,8696 & 97,99661 & 105,32561 \\
\hline Percepción & 4,2217 & 0,07853 & 4,06775 & 4,37558 \\
\hline
\end{tabular}


externa (Lafrancesco, 2004). En este caso, las dos pruebas tienen en común la disciplina y las competencias del profesional de Enfermería en Colombia.

Relación que existe entre la nota final de los Programas Académicos, resultados ECAES y la percepción de las estudiantes frente al logro de las competencias en el área profesional especifica (Gráfica 1). Se observa que existe correlación entre las notas de los programas académicos y los resultados ECAES, es decir, el estudiante con buen desempeño académico obtiene buenos resultados en la prueba ECAES; sin embargo, al relacionar estos dos elementos con la percepción, se observa que continúa siendo mayor la percepción del logro alcanzado por el estudiante que la nota obtenida en su programa académico y el resultado de los ECAES.

La percepción de los estudiantes sobre el logro de competencias al finalizar su periodo de formación es relevante y esto se debe a la forma en que se desarrollan los programas académicos de Cuidado de Enfermería, desde el ejercicio integral del Cuidado, programados en un calendario académico de cuatro a seis semanas de conceptualización teórica y realidades simuladas (laboratorios) y de 12-14 semanas de ejercicio profesional real, con presencia de tutor, que involucra el desarrollo cognoscitivo del estudiante a partir de unos saberes mínimos necesarios, el desarrollo de destrezas y habilidades en la resolución de problemas al enfrentar un sujeto de cuidado, trascendiendo del sentido común a una estructuración coherente entre la construcción teórica y la acción.

Enfermería por ser una profesión eminentemente práctica ha involucrado de una manera no visible, lo que señala el discurso por competencias, como un proceso de estructuración entre el saber, el saber hacer y el ser.

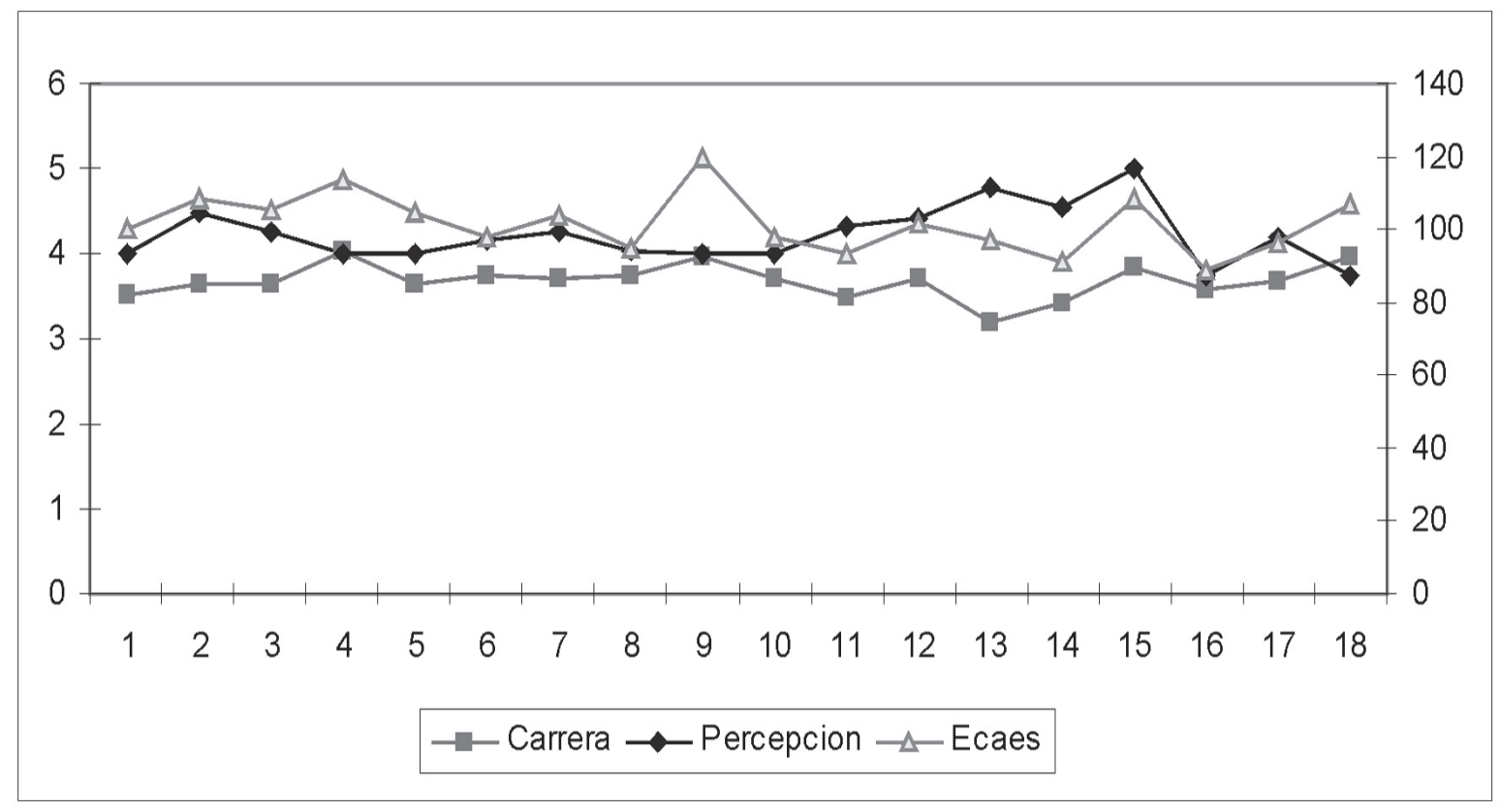

Gráfico 1. Relación entre la nota final de los cursos académicos, los resultados ECAES y la percepción de los estudiantes de séptimo semestre, frente al logro de las competencias en el área profesional específica.

\section{CONCLUSIONES}

Los estudiantes, en general, tienen la percepción de que los programas académicos de Cuidado, les ofrece elementos para afrontar el ejercicio profesional en las diferentes etapas del ciclo vital, pero las evaluaciones escritas internas y externas realizadas para valorar ese proceso no demuestran el alcance esperado, lo que permite establecer las debilidades que tienen los procesos escritos frente a la evaluación de las 
competencias logradas, en cuanto a habilidades instrumentales logradas por el estudiante.

La percepción de los estudiantes sobre el logro de competencias al finalizar su periodo de formación es relevante, esto se debe a la forma en que se desarrollan los programas académicos de Cuidado de Enfermería, desde el ejercicio integral del cuidado, con un abordaje amplio del componente práctico en donde se aplica claramente el constructivismo, donde sus experiencias forman parte de su adaptación al medio y la adaptación a los nuevos procesos, como es necesario cuando se enfrentan a situaciones extremas, que requieren de una toma de decisiones de carácter inmediato.

\section{RECOMENDACIONES}

El desarrollo de esta investigación deja muchas enseñanzas y un cúmulo interesante de información. El hecho que fuera realizada por los profesores que han participado en la construcción, desarrollo y análisis de las pruebas ECAES, condujo a que la investigación, en sí misma, se convirtiera en un espacio de constante reflexión y auto-evaluación del deber ser frente a una evaluación externa y masiva, que convierte al currículo en un ejercicio académico flexible y cambiante.

Se recomienda continuar la proyección de esta investigación, estableciendo los resultados con la percepción de los empleadores y el desempeño de los egresados para tener información más objetiva a cerca de las competencias alcanzadas por el ex alumno de la U.D.C.A.

\section{BIBLIOGRAFÍA}

ACOFAEN. 2003. Proyecto exámenes de estado de la calidad de la educación superior ECAES (documento de partida). Bogotá D.C., 11 de Junio de 2003. p.1-26.

ACOFAEN. 2005. 2004 - 2006. La Regionalización, estrategia de participación Boletín informativo, Bogota Abril - Mayo de 2.005.

CICARELLI, M. 2003, Actividades estrategias de enseñanza y aprendizaje, Editorial Tapa Blanca $1^{\mathrm{a}}$ ed., Barcelona. p.208.

FACULTAD DE ENFERMERÍA. 2006. Documento comparativo de análisis de resultados de ECAES 2003 - 2007. Noviembre 2006. Universidad de Ciencias Aplicadas y Ambientales U.D.C.A. p.6.

LAFRANCESCO, G. 2004. Acreditación de los centros educativos, evaluación institucional. Edit. Magisterio, 19ed. Bogotá. p.1-33.

MORENO, H. 2004. Hacia un currículo por competencias, ABC del educador. Ediciones SEM, Bogotá D.C., p.71; 43.

REPÚBLICA DE COLOMBIA. 2007. Desarrollo de programas académicos de educación superior. Decreto 2566 de 2003. Ley General de Educación 115 febrero 8 de 1994. p.475-498.

Recibido: Junio 6 de 2007

Aceptado: Abril 21 de 2008 